\title{
Species-Specific Effects of Cation Channel TRPM4 Small-Molecule Inhibitors
}

\author{
Prakash Arullampalam, Barbara Preti, Daniela Ross-Kaschitza, Martin Lochner, \\ Jean-Sébastien Rougier * and Hugues Abriel*
}

Swiss National Centre of Competence in Research (NCCR) TransCure, Institute of Biochemistry and Molecular Medicine, University of Bern, Bern, Switzerland

\section{OPEN ACCESS}

Edited by:

Anant Parekh,

University of Oxford, United Kingdom

Reviewed by:

Juliusz Ashot Kozak,

Wright State University, United States

Brij Bhan Singh,

The University of Texas Health Science Center at San Antonio, United States

*Correspondence: Jean-Sébastien Rougier jean-sebastien.rougier@

ibmm.unibe.ch

Hugues Abriel

hugues.abrie/@ibmm.unibe.ch

Specialty section: This article was submitted to Pharmacology of lon Channels and

Channelopathies,

a section of the journal Frontiers in Pharmacology

Received: 20 May 2021 Accepted: 30 June 2021

Published: 12 July 2021

Citation:

Arullampalam $P$, Preti $B$, Ross-Kaschitza D, Lochner M, Rougier J-S and Abriel H (2021) Species-Specific Effects of Cation

Channel TRPM4 SmallMolecule Inhibitors.

Front. Pharmacol. 12:712354. doi: 10.3389/fphar.2021.712354
Background: The Transient Receptor Potential Melastatin member 4 (TRPM4) gene encodes a calcium-activated non-selective cation channel expressed in several tissues. Mutations in TRPM4 have been reported in patients with different types of cardiac conduction defects. It is also linked to immune response and cancers, but the associated molecular mechanisms are still unclear. Thus far, 9-phenanthrol is the most common pharmacological compound used to investigate TRPM4 function. We recently identified two promising aryloxyacyl-anthranilic acid compounds (abbreviated CBA and NBA) inhibiting TRPM4. However, all aforementioned compounds were screened using assays expressing human TRPM4, whereas the efficacy of mouse TRPM4 has not been assessed. Mouse models are essential to investigate ion channel physiology and chemical compound efficacy.

Aim: In this study, we performed comparative electrophysiology experiments to assess the effect of these TRPM4 inhibitors on human and mouse TRPM4 channels heterologously expressed in TsA-201 cells.

Methods and Results: We identified striking species-dependent differences in TRPM4 responses. NBA inhibited both human and mouse TRPM4 currents when applied intracellularly and extracellularly using excised membrane patches. CBA inhibited human TRPM4, both intracellularly and extracellularly. Unexpectedly, the application of CBA had no inhibiting effect on mouse TRPM4 current when perfused on the extracellular side. Instead, its increased mouse TRPM4 current at negative holding potentials. In addition, CBA on the intracellular side altered the outward rectification component of the mouse TRPM4 current. Application of 9-phenanthrol, both intracellularly and extracellularly, inhibited human TRPM4. For mouse TRPM4, 9-phenanthrol perfusion led to opposite effects depending on the site of application. With intracellular 9phenanthrol, we observed a tendency towards potentiation of mouse TRPM4 outward current at positive holding potentials.

Conclusion: Altogether, these results suggest that pharmacological compounds screened using "humanised assays" should be extensively characterised before application in vivo mouse models.

Keywords: TRP channels, TRPM4, 9-phenanthrol, comparative physiology, mouse models, patch-clamp 


\section{INTRODUCTION}

Transient Receptor Potential (TRP) ion channels constitute a superfamily of cationic channels permeable to $\mathrm{Na}^{+}, \mathrm{Ca}^{2+}$ and/or $\mathrm{Mg}^{2+}$ (Nikolaev et al., 2019). By contributing to intracellular $\mathrm{Ca}^{2+}$ signalling (Tsagareli and Nozadze, 2020; Wang et al., 2020), they are critically involved in various calcium-dependent cell functions. The TRP family member TRPM4 has received much attention due to its implication in many physiological functions such as cardiac activity (Guinamard and Bois, 2007; Liu et al., 2010; Stallmeyer et al., 2012; Guinamard et al., 2015; Piao et al., 2015), immune response (Launay et al., 2004; Barbet et al., 2008; Hemmer et al., 2015), cancer (Sagredo et al., 2018), arterial constriction (Earley et al., 2004), insulin secretion (Cheng et al., 2007), and cell death (Simard et al., 2012). TRPM4 is expressed in many organs, including the heart, kidney, and brain (Reading and Brayden, 2007; Abriel et al., 2012; Simard et al., 2013; Flannery et al., 2015; Mehta et al., 2015), and is upregulated in specific cancer cells, rendering it a promising therapeutic target (Berg et al., 2016; Gao and Liao, 2019; Sagredo et al., 2019). Unlike most other TRP channels, which are calciumpermeable, TRPM4 is only permeable to monovalent cations, while intracellular calcium activates the channel in a voltage-dependent manner. Its activation leads to plasma membrane depolarisation, thereby regulating calcium homeostasis by decreasing the driving force for calcium to enter the cell through calcium-permeable channels (Launay et al., 2002; Nilius et al., 2004).

Many pre-clinical in vivo pharmacological studies are performed in mouse models, but TRPM4-inhibiting compounds are generally screened using heterologous systems expressing the human TRPM4 channel. It is highly relevant to study the effect of TRPM4-inhibiting compounds on human and mouse TRPM4, as TRP channel orthologues from different species may show remarkable functional differences: Mammalian TRPV1, for instance, is activated by capsaicin, whereas chicken trpv1 is entirely insensitive to it (Jordt and Julius, 2002; Chu et al., 2020). Another example is the speciesdependent effect of CMP1, a thioaminal-containing compound that activates rat TRPA1 but blocks human TRPA1 (Chen et al., 2008). Similarly, caffeine activates mouse TRPA1, but suppresses human TRPA1 (Nagatomo and Kubo, 2008). Other studies have shown species-specific effects of protein modulators and temperature on TRPM8 (Hilton et al., 2018) and TRPV1 (Papakosta et al., 2011), respectively.

In this study, we characterised the specificity and potency of three TRPM4 inhibitors (Figure 1A): 9-phenanthrol, 4-chloro-2[2-(2-chloro-phenoxy)-acetylamino]-benzoic acid (CBA, formerly named compound 5 in (Ozhathil et al., 2018)), and 4-chloro-2-(2-(naphthalene-1-yloxy) acetamido) benzoic acid (NBA, formerly named compound 6 in (Ozhathil et al., 2018)). Compound 9-phenanthrol has been widely used to inhibit TRPM4 in functional assays. However, its poor selectivity limits the validity of its use in animal models or primary cell lines (Burris et al., 2015; Ma et al., 2017). It is particularly problematic that past studies on TRPM4 have based their conclusions on patch-clamp assays that used this inhibitor despite its low potency and low specificity. CBA and NBA have been developed by our group and more potently inhibit TRPM4 than 9-phenantrol does (Ozhathil et al., 2018). Since these pharmacological compounds were discovered in a screening assay based on cell lines expressing human TRPM4, however, it was crucial to study their effect on mouse TRPM4.

Using the patch-clamp technique in cells expressing human or mouse TRPM4, we characterised the differences in specificity and potency of CBA, NBA, and 9-phenanthrol. We observed that CBA inhibits human TRPM4 but not mouse TRPM4, while NBA inhibits mouse and human TRPM4. Finally, 9-phenanthrol inhibits human TRPM4 and affects mouse TRPM4 currents differently depending on whether it is applied on the intracellular or extracellular side.

\section{MATERIALS AND METHODS}

\section{Cell Culture}

Transformed human embryonal kidney (TsA-201) cells stably overexpressing either mouse TRPM4, human TRPM4, or empty vector (pCDNA3.1 Zeo $(+)$ ) were generated using zeocin as a selection antibiotic. The cells were cultured in Dulbecco's Modified Eagle's medium (DMEM, \#41965039) (Invitrogen, Zug, Switzerland) supplemented with $10 \%$ FBS, $4 \mathrm{mM}$ glutamine, penicillin/streptomycin $(50 \mathrm{U} / \mathrm{ml})$, and Zeocin ${ }^{\circledR} 1 /$ 500 (Invitrogen cat\# R250-01). They were kept at $37^{\circ} \mathrm{C}$ with $5 \% \mathrm{CO}_{2}$ in a humidified atmosphere incubator.

\section{Western Blot}

To detect TRPM4 protein expression, whole-cell lysates were prepared by lysing cells in lysis buffer [50 mM HEPES pH 7.4; $150 \mathrm{mM} \mathrm{NaCl} ; 1.5 \mathrm{mM} \mathrm{MgCl}_{2}, 1 \mathrm{mM}$ EGTA pH 8.0; $10 \%$ glycerol; $1 \%$ Triton X-100; complete protease inhibitor cocktail (Roche, Mannheim, Germany)] for $1 \mathrm{~h}$ at $4^{\circ} \mathrm{C}$. Cell lysates were centrifuged for $15 \mathrm{~min}$ at $16,000 \times \mathrm{g}$ at $4^{\circ} \mathrm{C}$, pellets discarded, and protein concentration of the supernatant was evaluated using the Bradford assay. Sixty $\mu$ g of each protein sample was run on $9 \%$ polyacrylamide gels and transferred to a nitrocellulose membrane with the Turbo Blotdry blot system (Biorad, Hercules, CA, United States). After transfer, membranes were blocked with $0.1 \%$ BSA in PBS and incubated with rabbit primary anti-TRPM4 antibodies (epitope: VGPEKEQSWIPKIFRKKVC) (generated by Pineda, Berlin, Germany) diluted 1:750 in PBS/Tween using the SNAP i.d. system (Millipore, Billerica, MA, United States), followed by incubation with secondary antibodies (IRDye 800CW 1:1,000 in PBS/Tween, LI-COR Biosciences, Lincoln, NE, United States). Membranes were scanned using the LiCor Odyssey Infrared imaging system (LI-COR Biosciences).

\section{Compounds Tested}

Nine-phenanthrol (9-Phe) was obtained from Sigma-Aldrich (Buchs SG, Switzerland). Compounds CBA, NBA, and the other anthranilic acid amides (Figure 7) were synthesised as previously described (Ozhathil et al., 2018).

\section{Inside-Out Patch Clamp}

Electrophysiological recordings were performed in the insideout patch-clamp configuration with patch pipettes ( 1 and $2 \mu \mathrm{m}$ 
<smiles>Oc1cc2ccccc2c2ccccc12</smiles>

A (9-Phe)<smiles>O=C(COc1cccc2ccccc12)Nc1cc(Cl)ccc1C(=O)O</smiles>

4-Chloro-2-(2-(naphthalene-1 -yloxy)acetamido) benzoic acid (NBA)<smiles>O=C(COc1ccccc1Cl)Nc1cc(Cl)ccc1C(=O)O</smiles>

4-Chloro-2-[2-(2-chloro-phenoxy) -acetylamino]-benzoic acid (CBA)

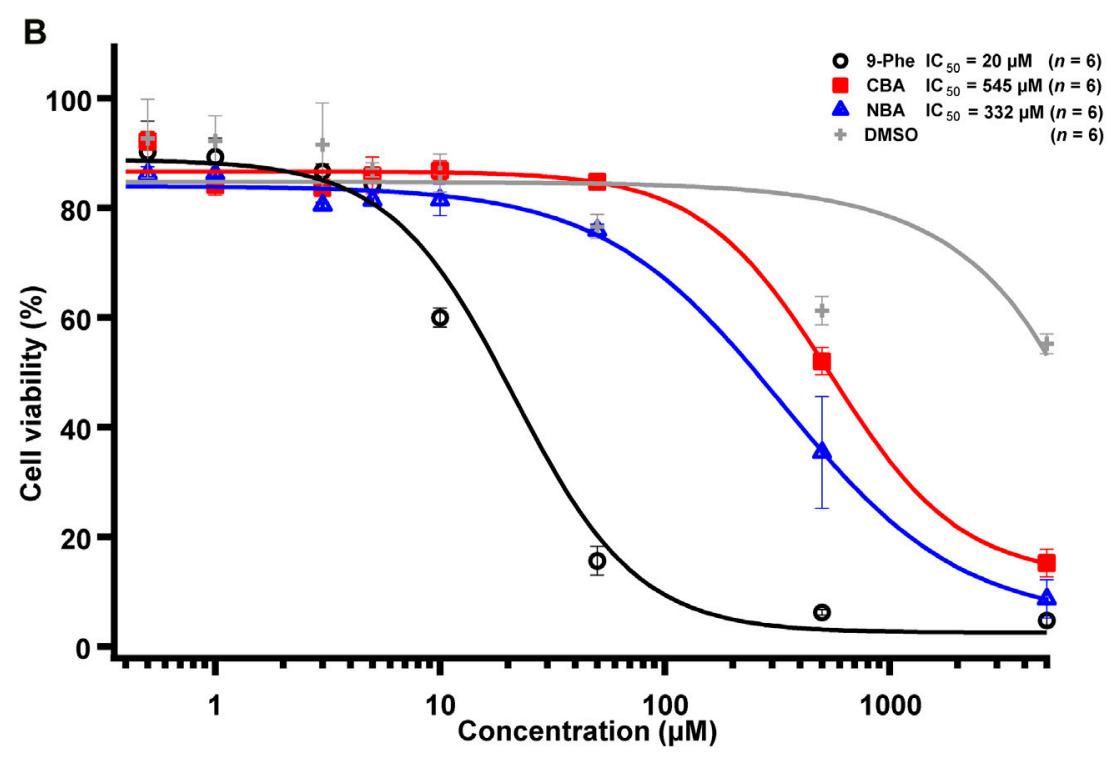

FIGURE 1 | TRPM4 pharmacological compounds used in this study. (A) Structure of 9-phenanthrol (left), NBA (middle), and CBA (right). (B) Concentration-response curve showing the cytotoxicity of 9-phenanthrol (black circles), CBA (red squares), NBA (blue triangles), and DMSO (grey plus symbols) ( $n=6$ for each concentration).

tip opening) pulled from $1.5 \mathrm{~mm}$ borosilicate glass capillaries (Zeitz-Instruments GmbH, München, Germany) using micropipette puller P 97 (Sutter Instruments, Novato, CA, United States). The tips were polished to have a pipette resistance of $2-4 \mathrm{M} \Omega$ in the bath solution. The pipette solution contained (in $\mathrm{mM}$ ) $150 \mathrm{NaCl}, 10 \mathrm{HEPES}$, and 2 $\mathrm{CaCl}_{2}$ (pH 7.4 with $\mathrm{NaOH}$ ). The bath solution contained (in $\mathrm{mM}) 150 \mathrm{NaCl}, 10$ HEPES, 2 HEDTA ( $\mathrm{pH} 7.4$ with $\mathrm{NaOH}$ ), and no $\mathrm{Ca}^{2+}$. Solutions containing $300 \mu \mathrm{M} \mathrm{Ca}^{2+}$ were made by adding the appropriate concentration of $\mathrm{CaCl}_{2}$ without buffer to a solution containing (in mM) $150 \mathrm{NaCl}, 10$ HEPES (pH 7.4 with $\mathrm{NaOH}$ ) as reported previously (Zhang et al., 2005). Bath solution with 0 and $300 \mu \mathrm{M} \mathrm{Ca}^{2+}$ concentrations were applied to cells by a modified rapid solution exchanger (Perfusion FastStep SF-77B; Warner Instruments Corp. CT, United States). Membrane currents were recorded with a Multiclamp 700B amplifier (Molecular Devices, Sunnyvale CA, United States) controlled by Clampex 10 via Digidata 1332A (Molecular Devices, Sunnyvale, CA, United States). Data were low-pass filtered at $5 \mathrm{kHz}$ and sampled at $10 \mathrm{kHz}$. Experiments were performed at room temperature $\left(20-25^{\circ} \mathrm{C}\right)$. The holding potential was $0 \mathrm{mV}$. For measuring steady-state currents, the stimulation protocol consisted of two sweeps with a total duration of $1,000 \mathrm{~ms}$, the first sweep at $-100 \mathrm{mV}$ for $500 \mathrm{~ms}$ and the second at $+100 \mathrm{mV}$ for $500 \mathrm{~ms}$ (Figure 2). The effect of the compounds on TRPM4 current has been calculated by averaging the last $100 \mathrm{~ms}$ of the second sweep at $+100 \mathrm{mV}$. The I-V relationships (IV curves) have been determined using an $\mathrm{I}-\mathrm{V}$ protocol from $-100 \mathrm{mV}$ to $+100 \mathrm{mV}$ for $500 \mathrm{~ms}$ with a difference of $20 \mathrm{mV}$ between each sweep (cf. Figure 6). The IV curves have been normalised to the maximum current. No fit was applied.

\section{Application of the Compounds and ATP}

After obtaining inside-out membrane patches, the presence of TRPM4 in the patch membrane was confirmed by switching the perfusion solution without calcium at the intracellular side of the membrane to a solution containing $300 \mu \mathrm{M}$ calcium, which activates TRPM4 and triggers the current. Once the TRPM4 currents were activated and stabilised for intracellular compound application, the perfusion solution containing $300 \mu \mathrm{M}$ calcium and either the compound or free adenosine triphosphate (ATP) was applied. For extracellular application, the compounds were added to the intracellular (pipette) solution. 


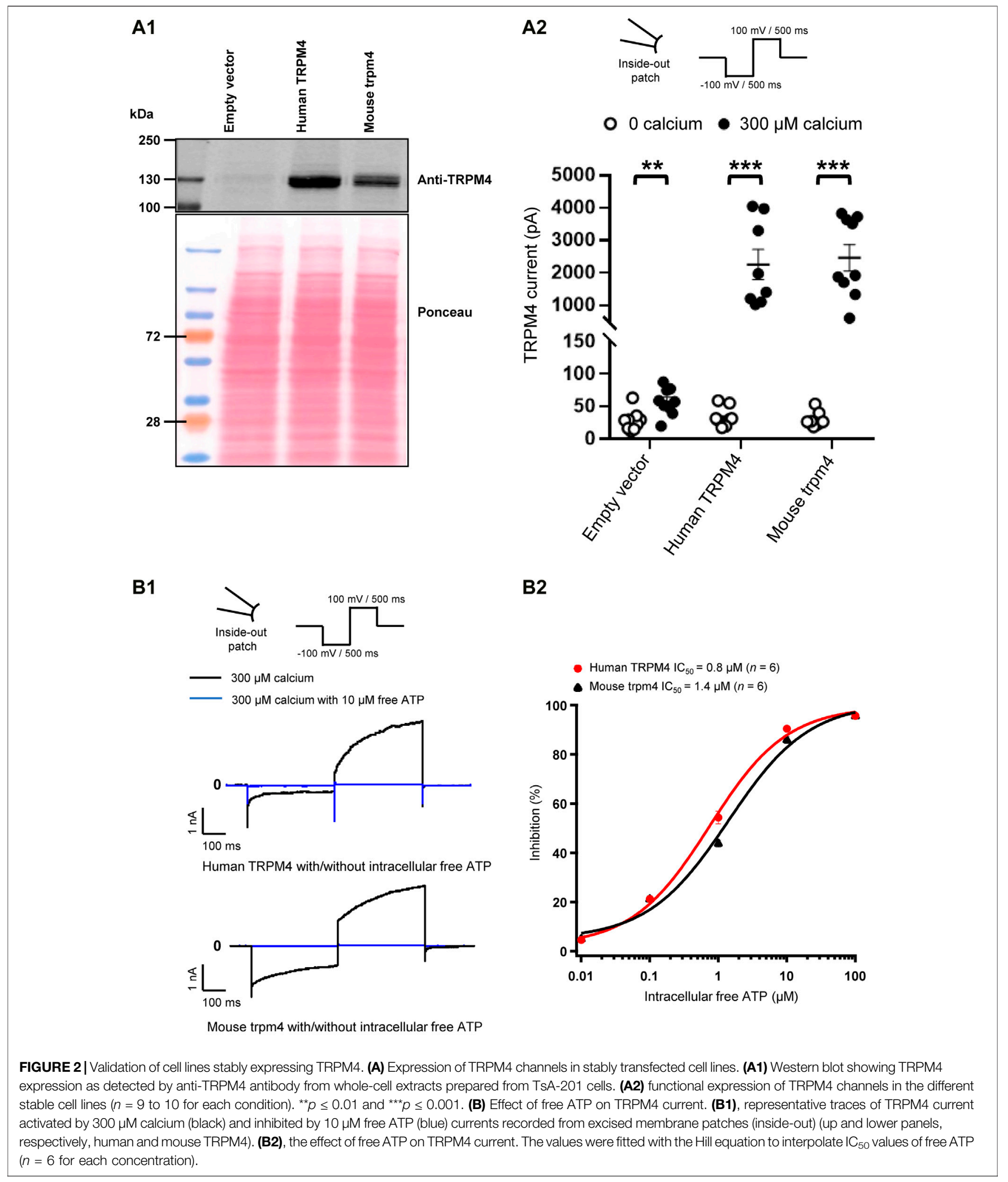

\section{Cytotoxicity Test}

Untransfected TsA-201 cells were seeded in a 96-wells plate at a concentration of 10,000 cells per well with DMEM and incubated at $37^{\circ} \mathrm{C}$ and $5 \% \mathrm{CO}_{2}$ in a humidified atmosphere incubator overnight. After reaching $80-90 \%$ of cell confluence, the cells in the 96-well plate were treated with different compound concentrations $(0.5,1,2,4,10,50$, 


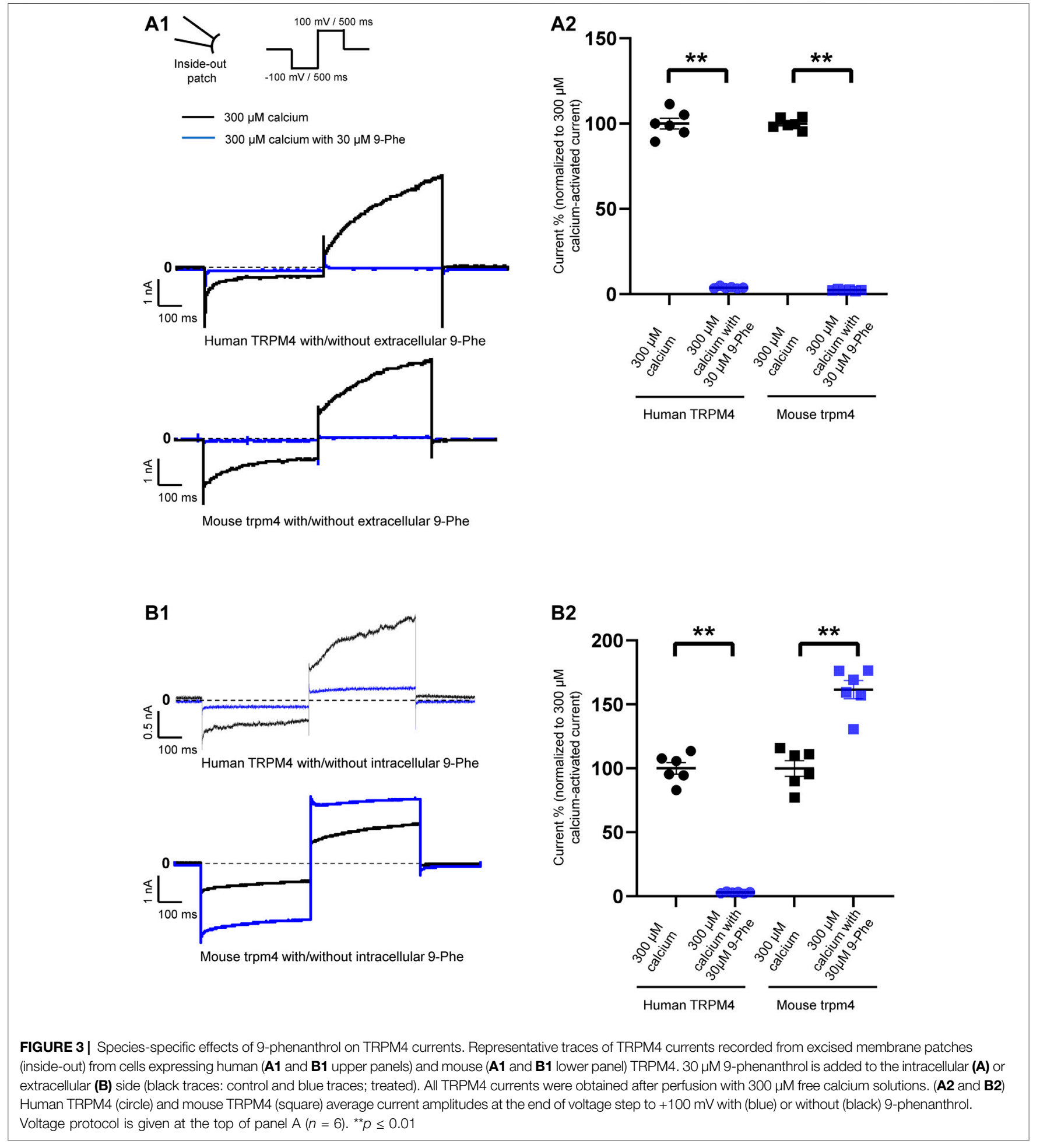

500, and 5,000 $\mu \mathrm{M}$ of CBA, NBA, and 9-phenanthrol) for $24 \mathrm{~h}$. After incubation, the cells were washed and incubated in $0.01 \%$ trypsin-EDTA until the cellular layer was completely detached. Cells were centrifuged down, and the pellet resuspended in $1 \mathrm{ml}$ PBS. Cells were then diluted in 1 part of $0.4 \%$ trypan blue and 1-part cell suspension. This mixture was incubated for $3 \mathrm{~min}$ at room temperature. Finally, the unstained (viable) and stained (nonviable) cells were counted in the haemocytometer (BRAND $^{\circledR}$ counting chamber BLAUBRAND ${ }^{\circledR}$, Wertheim, Germany).

\section{Data Analyses and Statistics}

Electrophysiology data were analysed using IGOR PRO 6 (WaveMetrics, Lake Oswego, United States), Normalized 


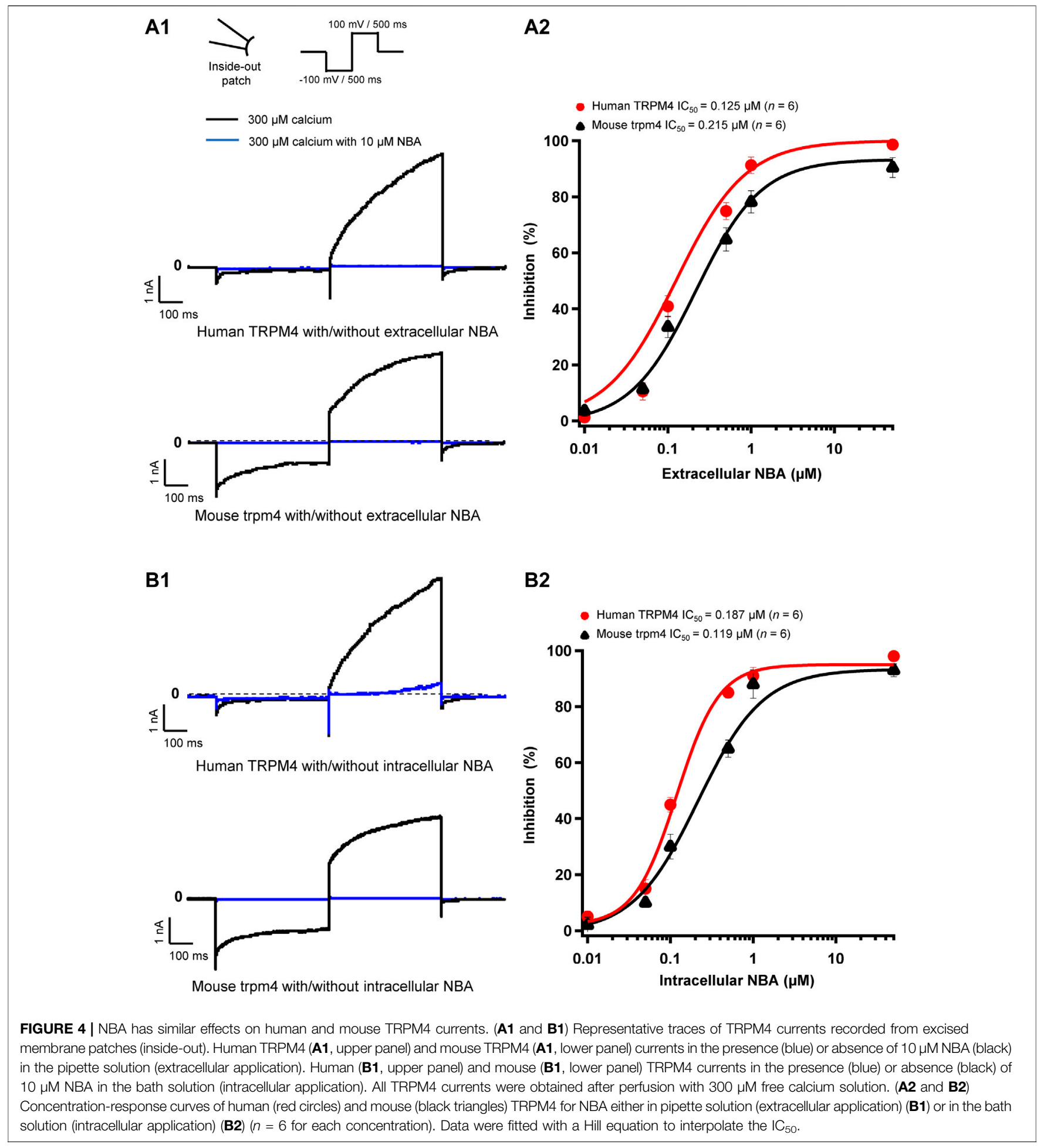

current percentage inhibition was calculated as follows ([activated current at $300 \mu \mathrm{M}$ calcium] - [activated current at $300 \mu \mathrm{M}$ calcium with compound] $) * 100 /$ [activated current at $300 \mu \mathrm{M}$ calcium]. Concentration-response curves were fitted using the Hill equation fit parameter of IGOR $\left(\mathrm{NC}=\mathrm{NC}_{\max }[\mathrm{Cmpd}] \mathrm{nH} /([\mathrm{Cmpd}] \mathrm{nH}+\right.$ EC50 $\mathrm{nH})$ ) where $\mathrm{NC}$ is normalized current [Cmpd] is compound concentration and $\mathrm{nH}$ is the Hill coefficient. Data are presented as mean \pm SEM except for Figures 3 and 7, where the data have been normalised to 100 for the current recorded in the presence of $300 \mu \mathrm{M}$ calcium only. Statistically significant differences conditions were determined using a non-parametric test with a Mann-Whitney post-test. $P$-values $\leq 0.05 ; \leq 0.01 ; \leq 0.001$ were 
considered as statistically significant and marked with ${ }^{*},{ }^{* *}$, and ${ }^{* * *}$ respectively in the different figure panels.

\section{RESULTS}

\section{Nine-Phenanthrol is More Cytotoxic Than CBA and NBA}

First, we tested the cytotoxicity of the three selected TRPM4 compounds (Figure 1A). The calculated $\mathrm{EC}_{50}$, corresponding to the concentration of the inhibitor required to induce $50 \%$ of cell death, was 15- to 25-fold lower for 9-phenanthrol ( $\left.\mathrm{EC}_{50} \sim 20 \mu \mathrm{M}\right)$ than for NBA $\left(\mathrm{EC}_{50} \sim 332 \mu \mathrm{M}\right)$ and $\mathrm{CBA}\left(\mathrm{EC}_{50} \sim 545 \mu \mathrm{M}\right)$, respectively (Figure 1B). These observations illustrate that the two new compounds CBA and NBA, are less cytotoxic than 9-phenanthrol.

\section{Characterisation of TsA-201 Cell Lines Stably Overexpressing Either Human or Mouse Transient Receptor Potential Melastatin Member 4}

To characterise the TsA-201 cell lines stably overexpressing either wild-type human or mouse TRPM4 generated for this study, we first tested the TRPM4 protein expression by western blot (Figure 2A1). Using an antibody recognising TRPM4 from both species, a robust expression of TRPM4 was observed in these modified cells compared to the cells transfected with an empty vector (Figure 2A1). To investigate if these cell lines express functional channels, we performed inside-out patch-clamp experiments to first record the calcium-sensitive current (Figure 2A2) and second to investigate the well-known TRPM4 inhibitory effect of free ATP on TRPM4 currents (Figures 2B1,B2). As expected, robust TRPM4 currents were observed in TsA-201 cell lines stably expressing either human or mouse TRPM4 channels compared to untransfected cells, also known to expressed endogenous TRPM4 channels (Figure 2A2). This current decreased after intracellular application of free ATP (Figures 2B1,B2). On the concentration-response curves depicted in Figure 3B2, the intracellular ATP $\mathrm{IC}_{50}$ for both types of channels was $0.8 \mu \mathrm{M}$ for human TRPM4 and $1.4 \mu \mathrm{M}$ for mouse TRPM4 (Figure 2B2). Altogether, these results confirm that both cell lines expressed functional human or mouse TRPM4 wild-type channels and can be used for further investigations.

\section{Nine-Phenanthrol Inhibits Human Transient Receptor Potential Melastatin Member 4 Current and Upregulates Mouse Transient Receptor Potential Melastatin Member 4 Current When Applied Intracellularly}

Knowing that lipophilicity tools have predicted that 9phenanthrol may cross the plasma membrane, we decided to record mouse and human TRPM4 currents in the presence of the three compounds (9-phenanthrol, CBA, and NBA) either applied at the intracellular side (bath solution) or the extracellular side (pipette solution).

First, we tested the effects of 9-phenanthrol on human and mouse TRPM4 channels. Thirty $\mu \mathrm{M}$ 9-phenanthrol, corresponding to the previously determined $\mathrm{IC}_{50}$ (Figure 1B), was applied using a perfusion solution containing $300 \mu \mathrm{M}$ calcium (Ozhathil et al,, 2018). Nine-phenanthrol inhibited human TRPM4 sodium current with a similar efficacy independently of the application side (extracellular or intracellular) (Figures 3A1,B1,A2,B2). Unexpectedly, while extracellular (pipette solution) 9-phenanthrol decreased the mouse TRPM4 current, it increased the current when applied intracellularly (Figures 3A1,B1,A2,B2). This increase was observed only in the presence of $300 \mu \mathrm{M}$ calcium, suggesting that 9phenanthrol acts as a potentiator rather than as an activator of the mouse TRPM4 current (Figure 8A). Moreover, this effect was more pronounced with low concentrations $(0.1 \mu \mathrm{M})$ of 9-phenanthrol than with higher concentrations (Figure 8B). When applying $100 \mu \mathrm{M}$ 9-phenanthrol, the observed potentiator effect was smaller than in the presence of $0.1 \mu \mathrm{M}$ 9-phenanthrol (Figure 8B). Altogether, these data illustrate that the effects mediated by 9-phenanthrol depend on the excised membrane patch application side and are species-specific.

\section{NBA Decreases Both Human Transient Receptor Potential Melastatin Member 4 and Mouse Transient Receptor Potential Melastatin Member 4 Currents}

Analogous to our experiments with 9-phenanthrol, we performed patch-clamp recordings using the compound NBA. We observed that $10 \mu \mathrm{M}$ NBA inhibited both human and mouse TRPM4 current independent of the side of the application (Figures 4A1,A2,B1,B2). The concentration-response curve for NBA revealed a sigmoid curve for both human and mouse TRPM4 currents (Figures 4A2,B2). Fitting the data with the Hill equation revealed that the $\mathrm{IC}_{50} \mathrm{~s}$ of NBA are 0.187 and $0.119 \mu \mathrm{M}$ for human and mouse TRPM4, respectively, when applied intracellularly (Figure 4B2). The $\mathrm{IC}_{50}$ s of extracellular NBA were 0.125 and $0.215 \mu \mathrm{M}$ with human and mouse TRPM4, respectively (Figure 4A2). Taken together, these data suggest that NBA does not discriminate between human and mouse TRPM4 channels and inhibits the TRPM4 current when applied to either side of the membrane.

\section{CBA Decreases Only Human, not Mouse, Transient Receptor Potential Melastatin Member 4 Currents}

Next, we assessed the effects of CBA on human and mouse TRPM4 currents. The application of $10 \mu \mathrm{M}$ CBA decreased the human TRPM4 currents independent of the side of the application (Figures 5A1,A2,B1,5B2). The $\mathrm{IC}_{50}$ s were $0.7 \mu \mathrm{M}$ (extracellular application/pipette solution) and $0.8 \mu \mathrm{M}$ (intracellular application) (Figures 5A2,B2). However, in membrane patches expressing mouse TRPM4 channels, neither the extracellular nor the intracellular application of CBA altered the TRPM4 currents (Figures 5A1,A2,B1,B2). Surprisingly, when CBA was applied intracellularly, a small but significant effect on mouse TRPM4 current was observed at positive voltages $(+100 \mathrm{mV})$, while at negative membrane voltages $(-100 \mathrm{mV})$, the current was significantly higher 


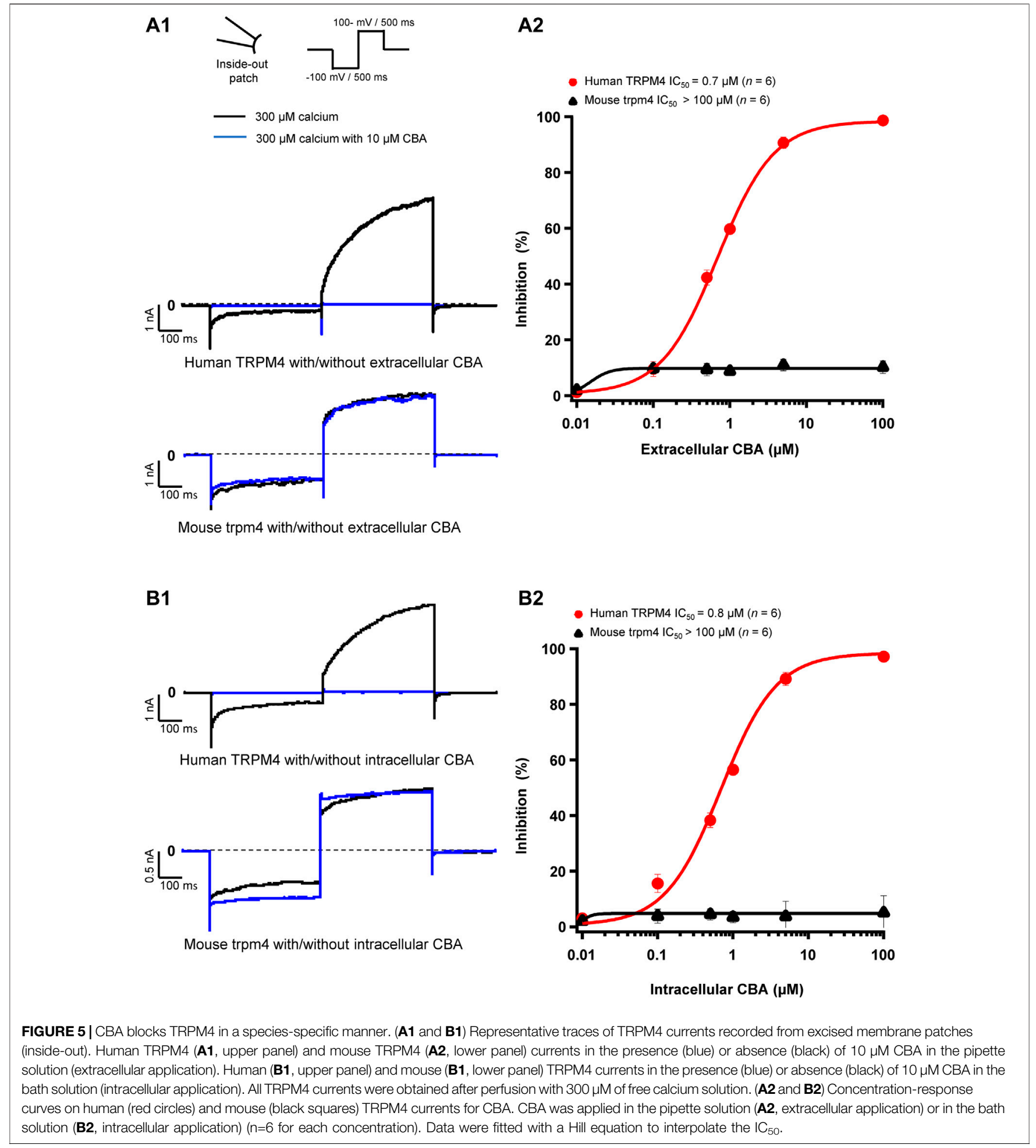

(Figure 5B1; Figure 6). In addition, the percentage of inactivation at $-100 \mathrm{mV}$ and activation at $+100 \mathrm{mV}$ were significantly decreased after CBA application (Table 1). Based on this observation, we recorded a current-voltage relationship (I-V) protocol to quantify the effect of intracellular CBA application on mouse TRPM4 current at different membrane voltages (Figure 6). As previously observed in Figure 5, CBA increased the inward current at negative membrane voltages (Figure 6) but only slightly altered the amplitude of the outward current at positives membrane voltages (Figure 6). Doing so, CBA led to a linearisation of the I-V relationship removing the rectification features. Altogether, these results 

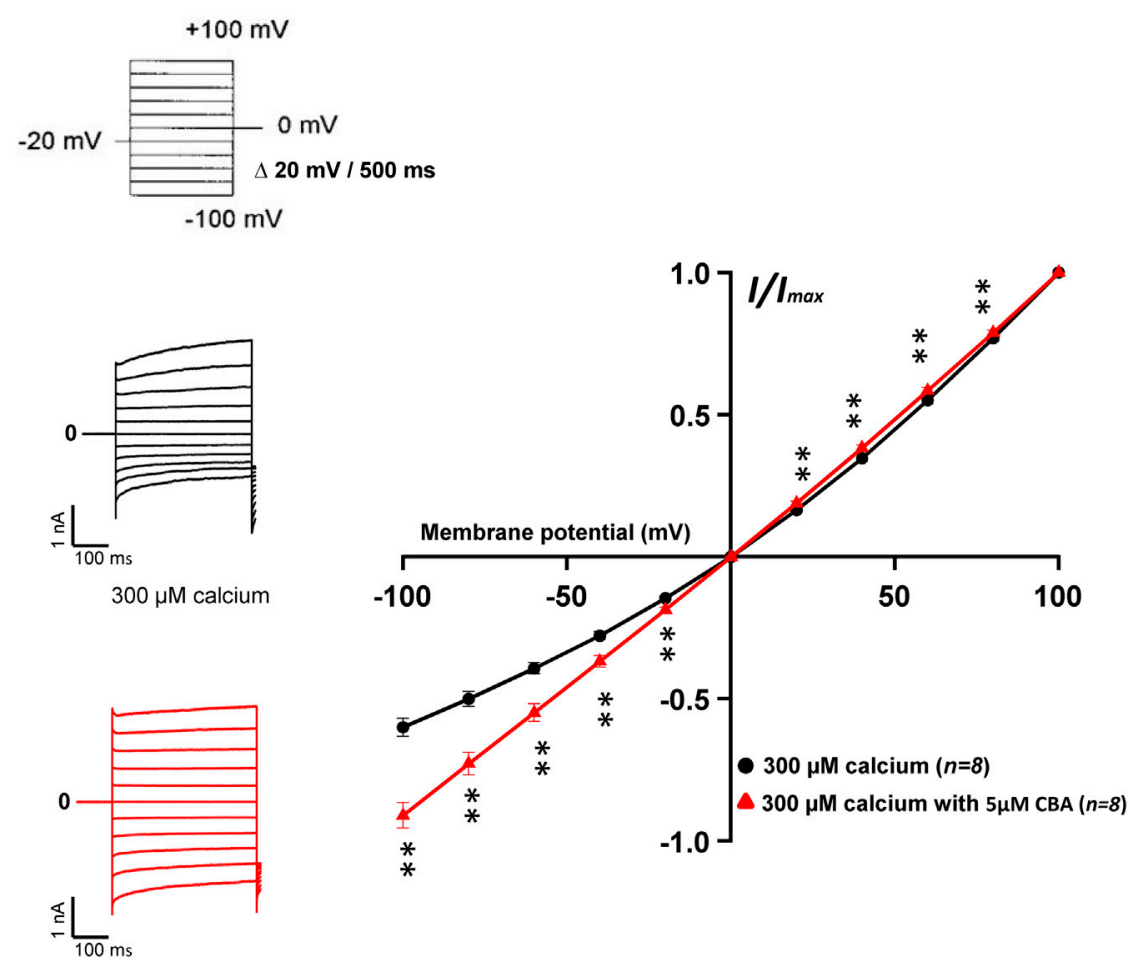

$300 \mu \mathrm{M}$ calcium with $5 \mu \mathrm{M}$ CBA

FIGURE 6 | Effects of CBA on mouse TRPM4 current. Left panel shows raw traces of mouse TRPM4 currents recorded using the step protocol depicted at the top of the figure, with (black) or without (red) $5 \mu \mathrm{M}$ CBA in the extracellular bath (intracellular application). Right panel shows IV curves, representing the average current amplitude at the end of each voltage step when the TRPM4 current was activated using $300 \mu \mathrm{M}$ free calcium solution (black) and treated with $5 \mu \mathrm{M}$ CBA (red) $(n=8)$. ${ }^{\star *} p \leq 0.01$

illustrate that the effect of CBA is species-specific, and CBA alters the inward current of mouse TRPM4.

\section{NBA- and CBA-Derived Compounds Have Similar, but not the Same, Effects}

To finally investigate if the effects observed could depend on further chemical structures, additional patch-clamp experiments were performed using compounds closely related to TRPM4 inhibitors CBA and NBA structures. They were selected based on a Structure-Activity Relationship (SAR) rationale (Ozhathil et al., 2018). The CBA-derived compound used was 4-chloro-2-(2-((4-chloro-2-methylphenoxy) propanamido) benzoic acid (CBA-d1) (Figure 7A1). The selected NBA-derived compounds were 4-chloro-2-(2-((4- chloronaphthalen-1-yl) oxy) acetamido) benzoic acid (NBA-d1) and 4-chloro-2-(2-((5,6,7,8tetrahydronaphthalen-1-yl) oxy) acetamido) benzoic acid (NBAd2) (Figures 7B1,C1). Interestingly, similarly to CBA, CBA-d1 did not decrease the mouse TRPM4 currents when applied intracellularly (Figures 7A1,A2). Contrary to CBA-d1, NBA-d1 and NBA-d2 could inhibit mouse TRPM4 currents, as also observed with NBA compounds (Figures 7B1,B2,C1,C2). Altogether, these results suggest that while some structurally related compounds behave similarly to the original one (NBA- $\mathrm{d} 1$ and NBA-d2 compared to NBA), some of them may have slightly different effects (CBA-d1 and CBA).

\section{DISCUSSION}

In this study, we assessed the species-specific effects of inhibitors of the calcium-activated non-selective monovalent ion channel TRPM4. Despite the relevance of TRPM4 for a wide range of physiological and pathological processes in different tissues, its roles remain not well understood. Identifying TRPM4 compounds that reliably block TRPM4 current in humans and mouse models is crucial to unravel the functions of TRPM4 and investigate it as a potential therapeutic target. We characterised the effect and species selectivity of three different TRPM4 small molecule inhibitors: 9-phenanthrol, NBA, and CBA. Further, we investigated three CBA and NBA derivatives. Nine-phenanthrol remains a widely used reference compound in studies to decipher the role of TRPM4 in mouse models. Although lipophilic prediction tools have suggested the capability of 9-phenanthrol to cross the plasma membrane, none of the studies published so far had investigated its effect when specifically applied on the intracellular side of the mouse TRPM4 channel using the inside out patch-clamp configuration. Here, we show for the first time 
TABLE 1 | Percentage of variation of mouse TRPM4 currents during the first $400 \mathrm{~ms}$ of the pulse at $-100 \mathrm{mV}$ and the first $400 \mathrm{~ms}$ of the pulse at $+100 \mathrm{mV}$ (see protocol Figure 3B1) with and without intracellular application of CBA at $10 \mu \mathrm{M}\left(n=6\right.$ for each parameter). ${ }^{* \star} p \leq 0.01$ and ${ }^{\star \star \star} p \leq 0.001$.

Without intracellular CBA Increase in percent of mouse TRPM4 current at $+100 \mathrm{mV}$ during the first $400 \mathrm{~ms}$
$-22 \pm 3 \%(n=11)$

$26 \pm 6 \%(n=11)$
With intracellular CBA $(10 \mu \mathrm{M})$
$-10 \pm 1 \%(n=11) * \star *$ $10 \pm 4 \%(n=11)$ ** that 9-phenanthrol inhibits mouse TRPM4 when applied on the extracellular part of the patch membrane, but its application on the inner membrane side leads to an evident potentiation of mouse TRPM4 current. Moreover, as suggested in Figure 8, the application of high concentrations of 9-phenantrol at the extracellular side of the cell may lead to a reduced-inhibition efficiency on TRPM4 current. It remains unclear whether 9phenanthrol has a direct effect on mouse TRPM4 or whether the observed increase in current is mediated by a second messenger such as Mg-ATP or phosphatidylinositol-4,5bisphosphate, which are known to restore the desensitisation of TRPM4 current (Zhang et al., 2005). Further experiments are required to address this question. Considering that 9-phenanthrol may cross the plasma membrane altogether, these observations raised the question of the global effect on the TRPM4 current of 9-phenanthrol would be when applied either in a mouse model or in cultured murine cells.

Interestingly, the potentiating effect of 9-phenanthrol when applied intracellularly to mouse TRPM4 was blunted at relatively high concentrations (Figure 8). Although more experiments are required to decipher the molecular mechanisms behind these observations, it can be proposed that 9-phenanthrol, due to its lipophilicity, when applied at a high concentration at the intracellular part of the cell, can partly cross the lipid bilayer reaching the extracellular side of the cell where it acts as an inhibitor. Conversely, 9phenanthrol applied to the outside surface of cells may also cross the plasma membrane and cause a potentiating effect at the intracellular-facing surface. Based on these considerations, published studies that used 9-phenanthrol should be interpreted with caution.

Considering that 1 ) the $\mathrm{IC}_{50}$ to block TRPM4 is much higher for 9-phenanthrol than for NBA and CBA, 2) 9phenanthrol partially block other ion channels (Abriel et al., 2012), and 3) its cytotoxicity is higher than NBA and CBA, our results strongly suggest that 9-phenanthrol is a suboptimal tool to dissect the TRPM4 physiological role in mice. It is therefore recommended to perform future experiments on mouse TRPM4 with NBA instead of 9phenanthrol. CBA has already been used in previous publications using cell lines expressing TRPM4 (Bianchi et al., 2018; Borgstrom et al., 2020). This compound is more potent and more specific than 9-phenanthrol on human TRPM4; indeed, CBA did not inhibit TRPV1, TRPV3, TRPV6, TRPM5, TRPM7, or TRPM8 (Ozhathil et al., 2018). Thus, CBA seems to be a promising inhibitor of human TRPM4, which is especially interesting for the cancer research field, where over-expression of human TRPM4 correlates with higher malignancy (Kappel et al., 2019; Borgstrom et al., 2020). CBA, however, does not inhibit the mouse TRPM4 current (Figure 5) and significantly decrease the activation and deactivation of mouse TRPM4 currents when applied intracellularly (Table 1). A possible explanation for this species-specific effect may lie in the $20 \%$ non-homology between mouse and human TRPM4 sequences. Generating chimeric constructs of mouse and human TRPM4 channels to address this hypothesis will be an important direction of study in the future. Moreover, cryo-EM structures of these channels in the absence or presence of NBA and CBA may provide important information concerning their TRPM4-binding site(s).

Interestingly and unexpectedly, CBA alters biophysical features of the mouse TRPM4 (outward and inward currents) (Figures 5, 6B1), suggesting different mechanisms of action of CBA on the mouse and human TRPM4, the latter being entirely inhibited by CBA. The site(s) of CBA action on the mouse and human TRPM4 is/ are not yet elucidated. The structure-function relationship explaining these observations thus remains unknown. CBAderived compounds inhibit neither inward nor outward mouse TRPM4 currents, suggesting that the differences in their structure may affect the biophysical properties of the channel differently. Taken together, our results suggest that CBA should only be used to study functional properties of human TRPM4 and avoided in mouse models.

Finally, NBA and the structurally related compounds efficiently inhibit both mouse and human TRPM4 channels (Figures 4, 7). NBA is, therefore, a novel and promising tool compound to study the role of TRPM4 in both humans and mice. Nonetheless, its site(s) of action still need(s) to be explored. We propose that characterising the channel properties of TRPM4 in diverse animal species and identifying the molecular determinants of TRPM4 pharmacological compounds will provide a foundation to develop new drugs to target TRPM4. To better characterise these compounds, it will be interesting to perform similar experiments in human in vitro models using either primary (native) cells such as human cardiomyocytes or induced pluripotent stem cell-derived cardiomyocytes. Lastly, both CBA and NBA should be tested in TRPM4 orthologs from other species.

The previous characterisation by TRPM4-mediated $\mathrm{Na}^{+}$ influx assays and electrophysiology experiments has shown that NBA is about 4-9 times more potent than CBA (Ozhathil 


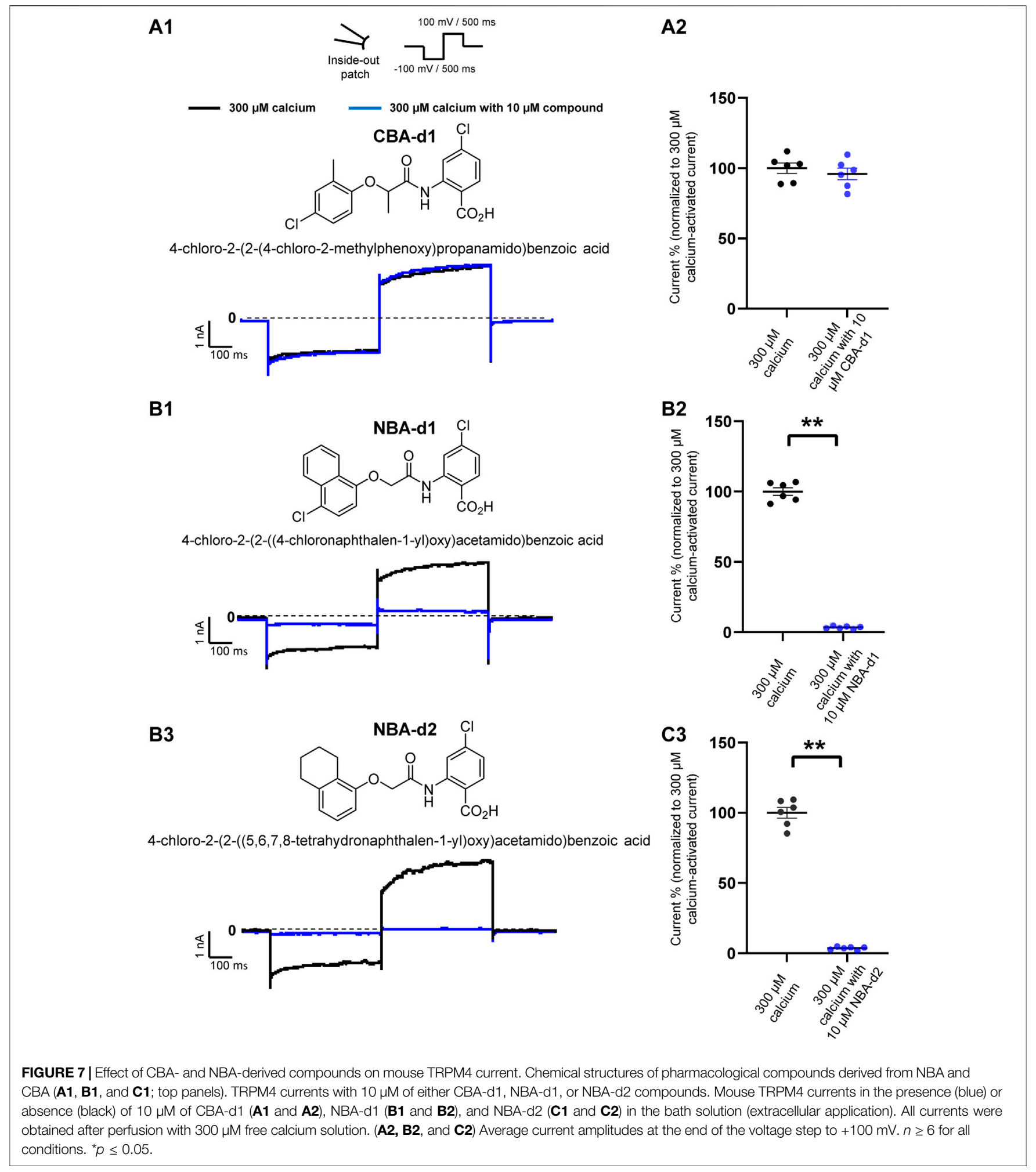

et al., 2018). Unfortunately, without a precise cryo-EM structure of the TRPM4 (Guo et al., 2017; Winkler et al., 2017) channel or other experimental evidence about the binding sites of $\mathrm{CBA}$ and $\mathrm{NBA}$, it is challenging to explain why NBA is more potent than CBA. We can only speculate that the additional ring of the naphthalene substituent of NBA can form attractive interactions with a putative hydrophobic pocket of TRPM4, which the smaller 

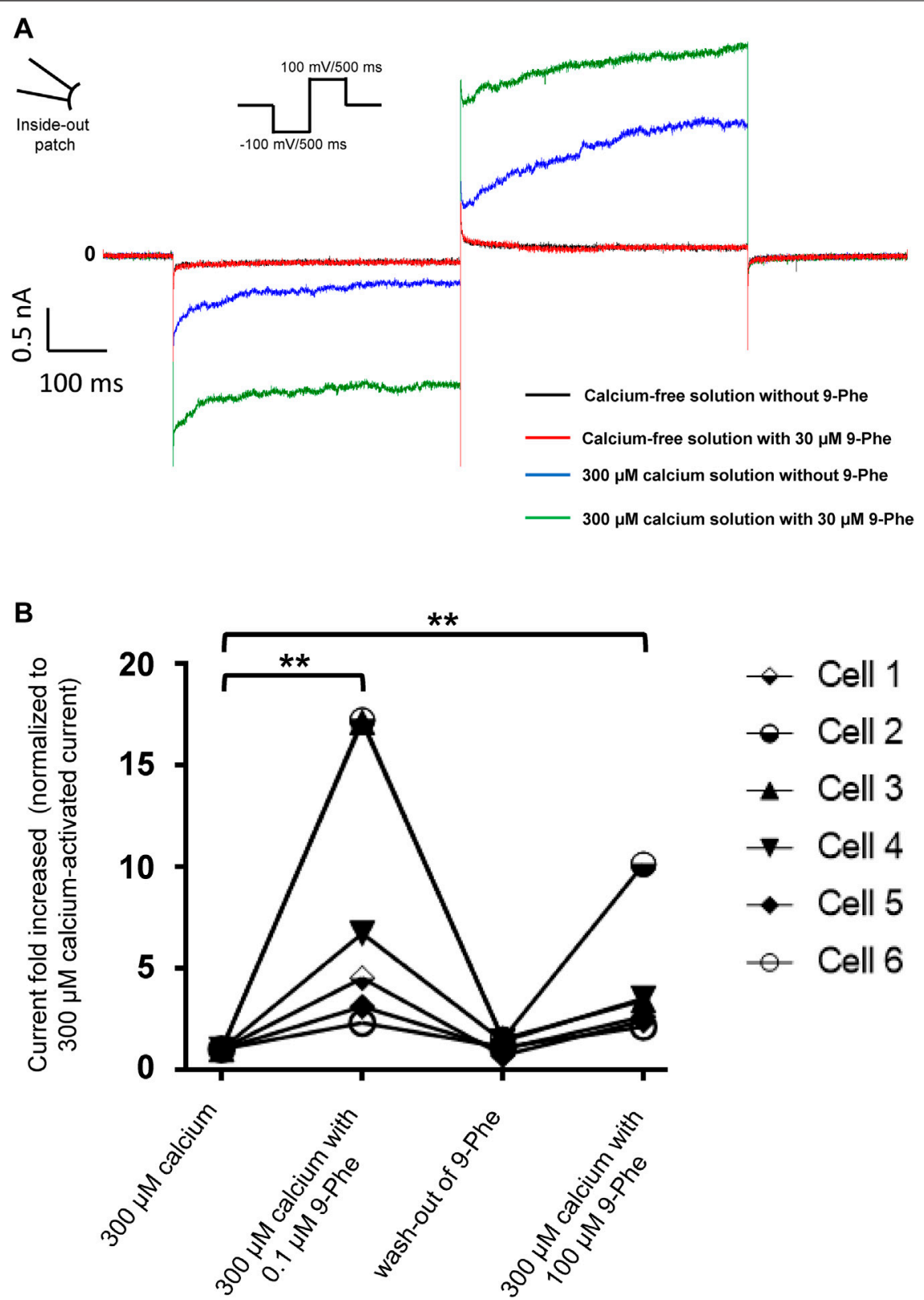

FIGURE 8| Nine-phenanthrol increases the mouse TRPM4 current only in the presence of calcium. (A) Representative traces of mouse TRPM4 currents recorded from excised membrane patches (inside-out) before and after application (intracellular application) of different solutions. Black trace: mouse TRPM4 current with a calcium-free and 9-phenanthrol-free solution; red trace: calcium-free and 9-phenanthrol solution with $30 \mu \mathrm{M}$ of 9-phenanthrol; blue trace: $300 \mu \mathrm{M}$ calcium solution without 9-phenanthrol; green trace: $300 \mu \mathrm{M}$ calcium and $30 \mu \mathrm{M}$ of 9-phenanthrol solution (similar observations were done with three different cells (data not shown). (B) Low concentrations of 9-phenanthrol potentiated more efficiently mouse TRPM4 currents compared to high concentrations. Normalised mouse TRPM4 currents from six different inside-out patches show a stronger potentiation of the current after low (0.1 $\mu \mathrm{M})$ 9-phenanthrol concentration application (extracellular application) compared to a high $(100 \mu \mathrm{M})$ concentration of this compound $(n=6)$.

2-chlorophenyl ring of CBA cannot, or only to a much smaller degree. Molecular docking of CBA and NBA into human and mouse TRPM4 could suggest potential binding sites that need to be corroborated by follow-up mutagenesis studies.

Overall, the findings of the present study suggest that new compounds should be carefully characterised before used as tool compounds in animal models to avoid misinterpretation of the results. Based on our work, NBA is a promising tool compound to study the role of the TRPM4 channel in normal physiology and under pathological conditions. Unfortunately, the other compound, CBA, seems to be only interesting for experiments using human TRPM4 (Ozhathil et al., 2018). Finally, the TRPM4 inhibitor 9-phenanthrol, which has 
been used for more than a decade to characterise TRPM4 function in different tissues, should be avoided as much as possible in favour of the two new characterised compounds NBA and CBA.

\section{DATA AVAILABILITY STATEMENT}

The original contributions presented in the study are included in the article/supplementary material, further inquiries can be directed to the corresponding authors.

\section{AUTHOR CONTRIBUTIONS}

PA, DR-K, and J-SR conceived and designed the experiments. PA, $\mathrm{BP}, \mathrm{DR}-\mathrm{K}$, collected, analysed, and interpreted the data. PA,

\section{REFERENCES}

Abriel, H., Syam, N., Sottas, V., Amarouch, M. Y., and Rougier, J.-S. (2012). TRPM4 Channels in the Cardiovascular System: Physiology, Pathophysiology, and Pharmacology. Biochem. Pharmacol. 84, 873-881. doi:10.1016/ j.bcp.2012.06.021

Barbet, G., Demion, M., Moura, I. C., Serafini, N., Léger, T., Vrtovsnik, F., et al. (2008). The Calcium-Activated Nonselective Cation Channel TRPM4 Is Essential for the Migration but Not the Maturation of Dendritic Cells. Nat. Immunol. 9, 1148-1156. doi:10.1038/ni.1648

Berg, K. D., Soldini, D., Jung, M., Dietrich, D., Stephan, C., Jung, K., et al. (2016). TRPM4 Protein Expression in Prostate Cancer: a Novel Tissue Biomarker Associated with Risk of Biochemical Recurrence Following Radical Prostatectomy. Virchows Arch. 468, 345-355. doi:10.1007/ s00428-015-1880-y

Bianchi, B., Smith, P. A., and Abriel, H. (2018). The Ion Channel TRPM4 in Murine Experimental Autoimmune Encephalomyelitis and in a Model of Glutamate-Induced Neuronal Degeneration. Mol. Brain 11, 41. doi:10.1186/ s13041-018-0385-4

Borgström, A., Hauert, B., Kappel, S., Zoni, E., Kiener, M., Stokłosa, P., et al. (2020). Small Molecular Inhibitors Block TRPM4 Currents in Prostate Cancer Cells, with Limited Impact on Cancer Hallmark Functions. J. Mol. Biol. 443, 166665. doi:10.1016/j.jmb.2020.09.024

Burris, S. K., Wang, Q., Bulley, S., Neeb, Z. P., and Jaggar, J. H. (2015). 9Phenanthrol Inhibits Recombinant and Arterial Myocyte TMEM16A Channels. Br. J. Pharmacol. 172, 2459-2468. doi:10.1111/bph.13077

Chen, J., Zhang, X.-F., Kort, M. E., Huth, J. R., Sun, C., Miesbauer, L. J., et al. (2008). Molecular Determinants of Species-specific Activation or Blockade of TRPA1 Channels. J. Neurosci. 28, 5063-5071. doi:10.1523/jneurosci.004708.2008

Cheng, H., Beck, A., Launay, P., Gross, S. A., Stokes, A. J., Kinet, J.-P., et al. (2007). TRPM4 Controls Insulin Secretion in Pancreatic $\beta$-cells. Cell Calcium 41, 51-61. doi:10.1016/j.ceca.2006.04.032

Chu, Y., Cohen, B. E., and Chuang, H. H. (2020). A Single TRPV1 Amino Acid Controls Species Sensitivity to Capsaicin. Sci. Rep. 10, 8038. doi:10.1038/ s41598-020-64584-2

Earley, S., Waldron, B. J., and Brayden, J. E. (2004). Critical Role for Transient Receptor Potential Channel TRPM4 in Myogenic Constriction of Cerebral Arteries. Circ. Res. 95, 922-929. doi:10.1161/ 01.res.0000147311.54833.03

Flannery, R. J., Kleene, N. K., and Kleene, S. J. (2015). A TRPM4-dependent Current in Murine Renal Primary Cilia. Am. J. Physiology-Renal Physiol. 309, F697-F707. doi:10.1152/ajprenal.00294.2015

Gao, Y., and Liao, P. (2019). TRPM4 Channel and Cancer. Cancer Lett. 454, 66-69. doi:10.1016/j.canlet.2019.04.012
DR-K, ML, J-SR, and HA drafted the manuscript and revised it critically for important intellectual content.

\section{FUNDING}

The research was funded by the Swiss National Science Foundation grant number 51NF40-185544 (NCCR TransCure, to HA and ML) and grant number 310030_184783 (to HA).

\section{ACKNOWLEDGMENTS}

We thank Dr. Lijo Cherian Ozhatil for providing suggestions and feedback on the manuscript. We acknowledge Dr. Sarah H. Vermij for proofreading and editing.

Guinamard, R., and Bois, P. (2007). Involvement of Transient Receptor Potential Proteins in Cardiac Hypertrophy. Biochim. Biophys. Acta (Bba) - Mol. Basis Dis. 1772, 885-894. doi:10.1016/j.bbadis.2007.02.007

Guinamard, R., Bouvagnet, P., Hof, T., Liu, H., Simard, C., and Sallé, L. (2015). TRPM4 in Cardiac Electrical Activity. Cardiovasc. Res. 108, 21-30. doi:10.1093/cvr/cvv213

Guo, J., She, J., Zeng, W., Chen, Q., Bai, X.-C., and Jiang, Y. (2017). Structures of the Calcium-Activated, Non-selective Cation Channel TRPM4. Nature 552, 205-209. doi:10.1038/nature24997

Hemmer, B., Kerschensteiner, M., and Korn, T. (2015). Role of the Innate and Adaptive Immune Responses in the Course of Multiple Sclerosis. Lancet Neurol. 14, 406-419. doi:10.1016/s1474-4422(14)70305-9

Hilton, J. K., Salehpour, T., Sisco, N. J., Rath, P., and Van Horn, W. D. (2018). Phosphoinositide-interacting Regulator of TRP (PIRT) Has Opposing Effects on Human and Mouse TRPM8 Ion Channels. J. Biol. Chem. 293, 9423-9434. doi:10.1074/jbc.ra118.003563

Jordt, S.-E., and Julius, D. (2002). Molecular Basis for Species-specific Sensitivity to "hot" Chili Peppers. Cell 108, 421-430. doi:10.1016/s0092-8674(02)00637-2

Kappel, S., Stokłosa, P., Hauert, B., Ross-Kaschitza, D., Borgström, A., Baur, R., et al. (2019). TRPM4 Is Highly Expressed in Human Colorectal Tumor Buds and Contributes to Proliferation, Cell Cycle, and Invasion of Colorectal Cancer Cells. Mol. Oncol. 13, 2393-2405. doi:10.1002/1878-0261.12566

Launay, P., Cheng, H., Srivatsan, S., Penner, R., Fleig, A., and Kinet, J. P. (2004). TRPM4 Regulates Calcium Oscillations after T Cell Activation. Science 306, 1374-1377. doi:10.1126/science.1098845

Launay, P., Fleig, A., Perraud, A.-L., Scharenberg, A. M., Penner, R., and Kinet, J.-P. (2002). TRPM4 Is a $\mathrm{Ca}^{2+}$-Activated Nonselective Cation Channel Mediating Cell Membrane Depolarization. Cell 109, 397-407. doi:10.1016/s0092-8674(02)00719-5

Liu, H., El Zein, L., Kruse, M., Guinamard, R., Beckmann, A., Bozio, A., et al. (2010). Gain-of-function Mutations in TRPM4 Cause Autosomal Dominant Isolated Cardiac Conduction Disease. Circ. Cardiovasc. Genet. 3, 374-385. doi:10.1161/circgenetics.109.930867

Ma, Z., Björklund, A., and Islam, M. S. (2017). A TRPM4 Inhibitor 9-Phenanthrol Inhibits Glucose- and Glucagon-like Peptide 1-Induced Insulin Secretion from Rat Islets of Langerhans. J. Diabetes Res. 2017, 5131785. doi:10.1155/2017/5131785

Mehta, R. I., Tosun, C., Ivanova, S., Tsymbalyuk, N., Famakin, B. M., Kwon, M. S., et al. (2015). Sur1-Trpm4 Cation Channel Expression in Human Cerebral Infarcts. J. Neuropathol. Exp. Neurol. 74, 835-849. doi:10.1097/nen.0000000000000223

Nagatomo, K., and Kubo, Y. (2008). Caffeine Activates Mouse TRPA1 Channels but Suppresses Human TRPA1 Channels. Proc. Natl. Acad. Sci. 105, 17373-17378. doi:10.1073/pnas.0809769105

Nikolaev, Y. A., Cox, C. D., Ridone, P., Rohde, P. R., Cordero-Morales, J. F., Vásquez, V., et al. (2019). Mammalian TRP Ion Channels Are Insensitive to Membrane Stretch. J. Cel Sci 132, jcs238360. doi:10.1242/jcs.238360

Nilius, B., Prenen, J., Janssens, A., Voets, T., and Droogmans, G. (2004). Decavanadate Modulates Gating of TRPM4 Cation Channels. J. Physiol. 560, 753-765. doi:10.1113/jphysiol.2004.070839 
Ozhathil, L. C., Delalande, C., Bianchi, B., Nemeth, G., Kappel, S., Thomet, U., et al. (2018). Identification of Potent and Selective Small Molecule Inhibitors of the Cation Channel TRPM4. Br. J. Pharmacol. 175, 2504-2519. doi:10.1111/ bph. 14220

Papakosta, M., Dalle, C., Haythornthwaite, A., Cao, L., Stevens, E. B., Burgess, G., et al. (2011). The Chimeric Approach Reveals that Differences in the TRPV1 Pore Domain Determine Species-specific Sensitivity to Block of Heat Activation. J. Biol. Chem. 286, 39663-39672. doi:10.1074/jbc.m111.273581

Piao, H., Takahashi, K., Yamaguchi, Y., Wang, C., Liu, K., and Naruse, K. (2015). Transient Receptor Potential Melastatin-4 Is Involved in HypoxiaReoxygenation Injury in the Cardiomyocytes. PLoS One 10, e0121703. doi:10.1371/journal.pone.0121703

Reading, S. A., and Brayden, J. E. (2007). Central Role of TRPM4 Channels in Cerebral Blood Flow Regulation. Stroke 38, 2322-2328. doi:10.1161/ strokeaha.107.483404

Sagredo, A. I., Sagredo, E. A., Cappelli, C., Báez, P., Andaur, R. E., Blanco, C., et al. (2018). TRPM4 Regulates Akt/GSK3- $\beta$ Activity and Enhances $\beta$-catenin Signaling and Cell Proliferation in Prostate Cancer Cells. Mol. Oncol. 12, 151-165. doi:10.1002/1878-0261.12100

Sagredo, A. I., Sagredo, E. A., Pola, V., Echeverría, C., Andaur, R., Michea, L., et al. (2019). TRPM4 Channel Is Involved in Regulating Epithelial to Mesenchymal Transition, Migration, and Invasion of Prostate Cancer Cell Lines. J. Cel Physiol. 234, 2037-2050. doi:10.1002/jcp.27371

Simard, C., Hof, T., Keddache, Z., Launay, P., and Guinamard, R. (2013). The TRPM4 Non-selective Cation Channel Contributes to the Mammalian Atrial Action Potential. J. Mol. Cell Cardiol. 59, 11-19. doi:10.1016/j.yjmcc.2013.01.019

Simard, J. M., Woo, S. K., and Gerzanich, V. (2012). Transient Receptor Potential Melastatin 4 and Cell Death. Pflugers Arch. - Eur. J. Physiol. 464, 573-582. doi:10.1007/s00424-012-1166-Z
Stallmeyer, B., Zumhagen, S., Denjoy, I., Duthoit, G., Hébert, J.-L., Ferrer, X., et al (2012). Mutational Spectrum in the $\mathrm{Ca}^{2+}$-Activated Cation Channel Gene TRPM4 in Patients with Cardiac Conductance Disturbances. Hum. Mutat. 33, 109-117. doi:10.1002/humu.21599

Tsagareli, M. G., and Nozadze, I. (2020). An Overview on Transient Receptor Potential Channels Superfamily. Behav. Pharmacol. 31, 413-434. doi:10.1097/ fbp.0000000000000524

Wang, H., Cheng, X., Tian, J., Xiao, Y., Tian, T., Xu, F., et al. (2020). TRPC Channels: Structure, Function, Regulation and Recent Advances in Small Molecular Probes. Pharmacol. Ther. 209, 107497. doi:10.1016/ j.pharmthera.2020.107497

Winkler, P. A., Huang, Y., Sun, W., Du, J., and Lü, W. (2017). Electron CryoMicroscopy Structure of a Human TRPM4 Channel. Nature 552, 200-204. doi:10.1038/nature24674

Zhang, Z., Okawa, H., Wang, Y., and Liman, E. R. (2005). Phosphatidylinositol 4,5bisphosphate Rescues TRPM4 Channels from Desensitization. J. Biol. Chem. 280, 39185-39192. doi:10.1074/jbc.m506965200

Conflict of Interest: The authors declare that the research was conducted in the absence of any commercial or financial relationships that could be construed as a potential conflict of interest.

Copyright (c) 2021 Arullampalam, Preti, Ross-Kaschitza, Lochner, Rougier and Abriel. This is an open-access article distributed under the terms of the Creative Commons Attribution License (CC BY). The use, distribution or reproduction in other forums is permitted, provided the original author(s) and the copyright owner(s) are credited and that the original publication in this journal is cited, in accordance with accepted academic practice. No use, distribution or reproduction is permitted which does not comply with these terms. 